\title{
Rehabilitation and physical activity for COVID-19 patients in the post infection period
}

\author{
Liska D, Andreansky M \\ Matej Bel University, Faculty of Arts, Department of Physical Education and Sports, Banska Bystrica, \\ Slovakia.david.liska27@gmail.com
}

\begin{abstract}
The first cases of COVID-19 were initially recorded in December 2019 in Wuhan, the capital of China's Hubei Province. The situation quickly escalated and turned into a global pandemic. COVID-19 is a highly infectious respiratory disease that leads to decreased respiratory, physical, and psychological function of affected patients (2). Patients' symptoms widely vary; from asymptomatic course to severe symptoms. Decrease in physical function, and, in some cases, a persistence of symptoms may be observed in patients, who overcame the infection period. Rehabilitation represents a potential treatment option for COVID-19 patients in post-infection period. Rehabilitation therapies may help to restore physical function in patients and to reduce the long-term effects of COVID19 infection (Ref. 37). Text in PDF www.elis.sk KEY WORDS: COVID19, rehabilitation, post-infection syndrome.
\end{abstract}

\section{Introduction}

The first cases of COVID-19 were reported in December 2019 in Wuhan, the capital of China's Hubei Province. The situation quickly turned into a global pandemic (1). Since the pandemic continues, there are limited data on clinical and prognostic factors in patients with COVID-19. COVID-19 is a highly infectious respiratory disease that leads to decreased respiratory, physical, and psychological function in affected patients (2). Patients' symptoms widely vary; from asymptomatic to severe (3). As the COVID-19 is highly infectious, the patients are isolated in order to limit the spread of SARSCoV-2. This leads to a significant reduction in social interactions, as a consequence of which the patients feel lonely and isolated (4). Mechanism causing pneumonia is particularly complex. It seems that the infection can elicit an excessive immune response in the host. COVID19 in some cases elicits a response generally known as 'cytokine storm', (5). In some cases, moreover, patients suffer from an extensive lung tissue inflammation. The main cytokine in this 'storm' is interleukin 6 (IL-6). IL-6 is produced by activated leukocytes and acts on a large number of cells and tissues. IL-6 assists in B cells differentiation.

Many patients remain lying in the intensive care unit for a longer period of time. Patients often remain in one position for several hours, which may lead, due to critical illness, to dysphagia, muscle weakness, myopathy and neuropathy, as well as to reduced mobility (6). In rare cases, the risk of falling may increase

Matej Bel University, Faculty of Arts, Department of Physical Education and Sports, Banska Bystrica, Slovakia

Address for correspondence D. Liska, Mgr, Matej Bel University, Faculty of Arts, Department of Physical Education and Sports, Banska Bystrica, Slovakia. due to muscle weakness. It may also result in walking problems potentially affecting patients' daily activities. In post infection period, patients may experience persistent pulmonary, musculoskeletal, neurological, cardiac, and psychological problems (7). These long-term effects can negatively affect patient's ability to perform daily activities and can also lead to social limitations. Rehabilitation treatment constitutes a potential treatment option for these patients. Rehabilitation therapies are based on individual needs of each patient (8). In order to determine the optimal rehabilitation approaches, the potential or existing effects of functional deficit in the post infection period patients should be assessed. Rehabilitation treatment is managed by a multidisciplinary team consisting of a physiatrist, physiotherapist, psychologist, and occupational therapist. Depending on the particular case, this rehabilitation therapy may be provided in several organizational modalities; in inpatient setting, outpatient setting, or home setting. Within the context of the COVID-19 pandemic, the use of remote monitoring and mobile intelligence technologies with wearable devices may enable the practice of intelligent, digital and remote rehabilitation (9-11).

Rehabilitation exercises may also prevent the occurrence of further complications (12). The elderly population is expected to be at risk of serious COVID-19-related complications. There is also an increased risk of serious complications in chronically ill and immunocompromised patients. An increased risk of mortality was found in patients with diabetes mellitus, chronic respiratory diseases, hypertension, and oncological diseases. A more severe course of the disease has been reported in the patients with underlying comorbidities, such as obesity (13). The most frequent triggers of serious complications in patients are respiratory failure, septic shock, or multiple organ dysfunction. A particularly serious complication is acute respiratory distress syndrome (ARDS). 
Mild disease is characterized by mild symptoms and absence of pneumonia signs. Rehabilitation after a mild disease can be provided on an outpatient basis by the means of telerehabilitation. Post-COVID19 patients may experience a persistence of symptoms even in the post-infection period. Chronic respiratory symptoms after the recovery from COVID-19 may include: shortness of breath on exertion, persistence of cough and wheezing, and chest pressure. However, these may or may not be related to exertion (14).

The negative effects associated with long-term intensive unit care are well described (15). Many patients suffer from postintensive care syndrome (PICS) after hospitalization in the intensive care unit. This syndrome causes a significant decline in muscle strength, physical performance, myopathy, and in individual cases neuropathy, loss of muscle mass, lack of nutrition, as well as an impaired emotional and physical health $(16,17)$. In addition to the post-intensive care syndrome, in the post-infection period patients also experience adverse symptoms. These include neuromuscular weakness, fatigue, decreased mobility, recurrent falls, deconditioning, as well as psychological disorders, such as anxiety, depression, poor concentration. Symptoms may persist for several months. Family members of patients may be similarly affected. There is a significant need in rehabilitation during the post-infection period $(18,19)$.

Rehabilitation treatment aims at improving patient's functional condition, resulting in an improved quality of life and a reduced physical disability. Physiatrists and physiotherapists play a key role in improving the functional effects of critical conditions, including long-term mechanical ventilation failure. They also play a key role in helping patients to recover from neuropathies and myopathies. Amid the COVID-19 pandemic, many physiatrists and physiotherapists are preparing for a substantial increase of patients with critical disease and respiratory-related functional disorders.

\section{Functional examination of patients in the post-infection period}

\section{Six Minute Walk Test}

The 6 minute walk test is a sub-maximal exercise test used to assess aerobic capacity and endurance in various chronic conditions. The distance covered in 6 minutes is used as a result to compare the changes in patients' functional capacity. This test can be used in patients of all ages. The test was initially designed to help in the assessment of patient with cardiopulmonary issues. Gradually, it was introduced in numerous other conditions. It also plays a potential role in the assessment of patients with COVID-19 in post-infection period. It assesses the functional capacity of an individual and provides valuable information on all systems during physical activity, including pulmonary and cardiovascular systems. To perform the test, it is necessary to measure the circle in which will patients perform the test. Another necessary equipment is a stopwatch. Sporttesters may be used to measure a pulse frequency.

\section{The Chair Stand Test}

Chair stand test is used for testing lower limb strength and endurance in older adults. It is a part of the Fullerton Functional
Fitness Test Battery (21). The chair stand test is used to assess a functional lower extremity strength, transitional movements, balance, and a fall risk in older adults (22). Scoring is based on the amount of time (to the nearest decimal in seconds). During the test, patient transfers from a seated to a standing position and back to sitting five times. The equipment needed in performing test includes stopwatch and a standard height chair with a straight back. In order to execute the test correctly, the test taker sits on the chair with their straight back resting against the chair back. The test taker is also instructed to fold their arms across their chest. Then the test taker should be instructed to do five sit-to-stands as quickly as possible. Another variant is the 30 -second chair stand test. It is performed in the same design as the first variant, but it involves recording the number of stands a person can complete in 30 seconds.

\section{Step Test}

The test was developed at Harvard University in 1942. It is also known as Harvard cardiac stress test (23). Step test is one of the oldest and most widely used cardiac stress tests. This test is also used in cardiac rehabilitation. The test is used to measure and assess an individual's circulatory and aerobic fitness and is based on the measurements of cardiac activity. One of the main indicators of cardiac activity is a change in heart rate. The measurement is based on the principle of a directly proportional relationship between cardiovascular fitness and the recovery rate of the heart rate to the initial values after a strenuous exercise. The metabolic demands of muscle tissue increase during a physical exercise, which leads to an increased cardiac activity as well. The better the individual's cardiovascular fitness, the sooner their heart rate values return to normal.

There are several versions of Harvard step test. In the most widely used version of the test, an individual steps up and down on the box at a rate of 30 steps per minute. Height of the box is $40 \mathrm{~cm}$ for men and $30 \mathrm{~cm}$ for women. Duration of the exercise is 5 minutes or until exhaustion. After finishing the exercise, the test taker lies down on their back and the total number of their heart beats are counted 1, 2, and finally 3 minutes after finishing the exercise. The patient immediately sits down on completion of the test, and the total number of their heart beats are counted from 1 to $1 \frac{1}{2}$ minutes after finishing and from 2 to $2 \frac{1}{2}$ minutes after finishing and finally from 3 to $3 \frac{1}{2}$ minutes after finishing. By using the measured data in formula, we subsequently obtain a certain index of physical proficiency, the fitness index. Calculation of fitness index:

(te) $=300$ seconds - time until exhaustion (in seconds)

$(\mathrm{hb})=$ sum of heartbeats after 1,2 , and 3rd minute

\section{Timed up and go test (TUG)}

This test is used predominantly in elderly population. It is designed to assess the functional ability of patients and determine the risk of falling. It may be beneficial in determining the physical impairment caused by the infection in elderly patients with COVID-19 after the resolution of the infectious period. Equipment needed to perform the test: chair and stopwatch. In order to 
perform the test correctly, patient starts in a seated position with their back resting against the chair back. Patient positions their feet on a place marked by a line. Patient stands up upon therapist's command and walks 3 meters. Therapist records the time needed to walk 3 meters and back to the chair. A longer distance can be used as a variation of the test.

\section{The post-COVID-19 functional status (PCFS)}

It is a simple tool to monitor the course of symptoms and the impact of symptoms of COVID-19 infection. PCFS scale may help to assess patients, who need a rehabilitation treatment after recovery from COVID-19 infection. It covers the full spectrum of functional outcomes and focuses on patients' limitations. Grade 0 reflects the absence of any functional limitation. Grade 1 reflects that the symptoms, pain or anxiety are present to an increasing degree. Grade 2 and 3 account for patient's impaired ability in performing various daily activities (24). Finally, grade 4 accounts for patients with severe functional limitations.

\section{Rehabilitation options}

Early active mobilization is important for improving muscle strength, which results in a better mobility in patients discharged from hospital, as well as in the improvement in patients' quality of life outside the hospital. An important part of rehabilitation includes a neuromotor mobilization together with a passive mobilization and an active exercises to maintain or improve the range of motion. Rehabilitation therapies may also help with algic manifestations in the post-infection period patients.

When determining the functional fitness of patient, it is important to take into account the level of physical activity in the home environment and to determine the preferred type of movement.

Type of exercise: e.g. gait, cycling, swimming, yoga, pilates, nordic walking, housework, gardening etc. $(25,26)$.

Duration: Duration of daily physical activity.

Frequency: Physical activity during the week.

Exercise Intensity: (light, moderate, or intense exercise).

\section{Types of exercises}

Muscle weakness may be present in post-COVID19 patients. Strength training is a physical exercise that uses resistance in order to stimulate muscle contraction. Strength training also leads to an improved joint mobility, as well as to an increased strength of muscles, tendons and ligaments (27). During the strength training, it is important to maintain a slow controlled movement. When it comes to resistance exercise, it is possible to regulate the load, taking into account the patient's health condition. Strength training including both concentric and eccentric muscle contraction is a safe, simple, and effective way to induce and increase muscle strength.

\section{Aerobic exercise}

It is the most common type of physical activity (28). Aerobic exercise is performed with a moderate intensity. The basic aero- bic exercises are gait, swimming, cycling, and nordic walking. In the post-infection period, it is important to start walking with a moderate gait, and then to increase the intensity. Interval exercises may also be applied, providing that the patient's health condition has sufficiently improved.

\section{Graded exercise}

After respiratory symptoms resolved, a potential problem for patients may be deconditioning and a reduced lung function. Graded exercise is based on a gradual increase of the intensity of physical activity. It has to be used accordingly to patient's individual physical ability. Exercise begins with a moderate intensity and is important in terms of improving cardiovascular function. One of the most basic types of exercise is gait. Gait should be initially taught at patient's natural pace and should not exceed $40-50 \%$ of patient's maximum heart rate (COVID-19). The increase of intensity must not occur before the infectious period has resolved. Physical activity should be performed at least 5 times a week for 30 minutes. If patient tolerates the load and an adequate physical activity leads to an improvement in symptoms, the intensity and duration of physical activity may be prolonged.

\section{Rehabilitation methods}

To improve patients' health, we can use also other rehabilitation methods. These exercises are most frequently applied to improve the posture and alleviate pain in patients. The most commonly used ones are yoga and pilates. Other rehabilitation methods may be used to improve musculoskeletal problems. Several forms of manual therapy might be used to reduce pain manifestations in the post-infection patients.

Another potential treatment option is occupational therapy. Occupational therapy can be suitable especially for elderly people, who have lost their autonomy and have limitations in performing their daily activities. It may speed up the homecoming and help to stimulate the need for independence. Another important rehabilitation component is psychological care. Psychological care is provided for patients with mental disorders, such as anxiety, depression, and post-traumatic stress disorder.

\section{Pulmonary rehabilitation}

Pulmonary rehabilitation focuses at improving functional condition in patients with lung diseases (29). An important part of treatment is pulmonary therapy. The aim of pulmonary rehabilitation in patients with COVID-19 is to improve the symptoms of dyspnoea, alleviate anxiety, reduce complications, minimize physical disability, as well as to maintain functioning and improve the quality of life.

Pulmonary rehabilitation involves breathing exercises based on assessment and monitoring of exercise tolerance with the aim of improving breathing control (30). Breathing exercises are used to improve the tidal volume and reduce psychological effects (stress, anxiety, and depression) $(31,32)$. Exercise training is the most important component of pulmonary rehabilitation. It can be initially performed by a lying patient, in case of significantly 
depressed patient even in an inpatient setting (patient lying on a hospital bad), and subsequently advance to gait in an outpatient setting.

Pulmonary rehabilitation is based on a comprehensive assessment of patient's health and various individualized techniques designed to prevent complications that may cause or worsen respiratory symptoms. Pulmonary rehabilitation also helps to recover from infectious diseases of the respiratory system. Additionally, it includes general exercises aimed at improving the physical fitness. Exercises to improve the function of the inspiratory muscles are an important part of the therapy. Pulmonary rehabilitation also focuses on the practice of coughing and the removal of airway secretions. Another important part of the therapy is a psychological component that includes the education of the patient. Pulmonary rehabilitation is a simple, effective, safe, and timesaving approach that leads to faster reconvalescence of patient. Within the assessment, it is important to focus on patient's functional ability, mobility, cardiovascular function, and on the assessment of breathing stereotype (frequency, amplitude).

Another type of exercise is an active cycle of breathing techniques. The active cycle of breathing techniques utilises the combinations and cycles of airway clearance techniques in order to clear the obstructed parts of lungs. Another option is autogenic drainage. Autogenous drainage is a common technique based on a combination of manoeuvres to mobilize and centralize secretions by short breaths in order to capture secretions in the peripheral airways. This is followed by normal breathing to capture secretions in the intermediate airways, then by a deep breath and a strong cough to induce excretion.

\section{The effectiveness of rehabilitation in regards to an improve- ment of patients' functional condition}

Exercise has a positive effect on systemic endothelial function and the inflammatory response (33). Regular exercises lead to significant strengthening of the immune system. Exercise have also a positive effect on apoptosis after the recovery from the infection.

Community-acquired pneumonia in the elderly population leads to decrease in daily activities (ADL) and quality of elderly patients' lives. It is accompanied with a decreased physical and mental functions. In elderly patients, who overcame COVID-19, it is important to improve pulmonary function, as it is an important factor in maintaining ADL and QoL.

The effect of pulmonary rehabilitation on pulmonary function and quality of life in older adults with COVID-19 was tested by Liu et al. (35). A total of 72 patients were randomly divided into experimental and control groups. The experimental group underwent a pulmonary rehabilitation and the control group did not. Patients underwent the pulmonary rehabilitation that lasted six weeks. After six weeks, there was recorded, based on comparison with control group, a significant improvement in respiratory function, a 6-minute walk test, and quality of life SF-36 score. The effectiveness of respiratory rehabilitation in an 80 -year-old patient was testes out by Shan et al. (36). Patient required an intubation for 14 days in the intensive care unit. Patient underwent 1 month rehabilitation after hospitalisation. Patient's gait distance, gait speed, heart rate variability, and oxygen saturation showed improvements.

Patients with COVID-19 are more likely to suffer from prevalent anxiety and depression. This can lead to sleep problems. The effectiveness of progressive muscle relaxation in patients with $\mathrm{CO}$ VID-19 was tested by Liu et al. (35). Progressive muscle relaxation is a method of deep muscle relaxation based on the notion that muscle tension is a physiological response of the human body to thinking. This technique was described by Jacobsen. Progressive muscle relaxation is easy to learn. It does not require a specific time and place, nor special technology or equipment. The aim of the study by Liu et al. (37) was to test the effectiveness of progressive muscle relaxation in patients with COVID-19 in regards to sleep quality and anxiety. A total of 51 patients were randomly divided into experimental and control groups. The experimental group underwent a progressive muscle relaxation for 30 minutes per day for 5 consecutive days. The control group did not undergo the therapy. In comparison to the control group, the anxiety and sleep quality scores of the experimental group after an intervention were statistically significant $(\mathrm{P}<0.001)$.

\section{Conclusion}

Rehabilitation represents a potential treatment option for patients after recovery from COVID-19 infection. Rehabilitation therapies can help to restore physical function in patients and alleviate the effects of COVID-19 infection.

\section{References}

1. EI Hassoun O, Valaskova Z, Polak S, Hulin I. Few insights on the problem of COVID-19. Bratisl Med J 2020; 121 (7): 471-474.

2. Hatala R. Proposal for complementary targeted public health antiepidemic measures during the pandemics of COVID-19. Bratisl Med J 2020; 121 (7): 459-461.

3. Zhu Y, Wang Z, Zhou Y, Onoda K, Maruyama H, Hu C et al. Summary of respiratory rehabilitation and physical therapy guidelines for patients with COVID-19 based on recommendations of World Confederation for Physical Therapy and National Association of Physical Therapy. J Phys Ther Sci 2020; 32 (8): 545-549.

4. Liska D, Poljak J. Possibilities of rehabilitation in corona virus disease (COVID-19). Rehabilitacia 2020; 57 (2): 78-90.

5. Yormaz B, Ergun D, Tulek B, Ergun R, Korez KM, Suerdem M et al. The evaluation of prognostic value of acute phase reactants in the COVID-19. Bratisl Med J 2020; 121 (9): 628-633.

6. Smondack P, Gravier F-É, Prieur G, Repel A, Muir J-F, Cuvelier A et al. [Physiotherapy and COVID-19. From intensive care unit to home care-An overview of international guidelines]. Rev Mal Respir 2020; 10.

7. Barker-Davies RM, O'Sullivan O, Senaratne KPP, Baker P, Cranley M, Dharm-Datta $\mathbf{S}$ et al. The Stanford Hall consensus statement for post-COVID-19 rehabilitation. Br J Sports Med 2020; 54 (16): 949-959.

8. Asly M, Hazim A. Rehabilitation of post-COVID-19 patients. Pan Afr Med J 2020; 36: 168.

9. Nakayama A, Takayama N, Kobayashi M, Hyodo K, Maeshima N, Takayuki $\mathbf{F}$ et al. Remote cardiac rehabilitation is a good alternative of 
310-314

outpatient cardiac rehabilitation in the COVID-19 era. Environ Health Prev Med 2020; 25 (1): 48.

10. Salawu A, Green A, Crooks MG, Brixey N, Ross DH, Sivan M. A Proposal for Multidisciplinary Tele-Rehabilitation in the Assessment and Rehabilitation of COVID-19 Survivors. Int J Environ Res Public Health 2020; 17 (13).

11. Urban M, Kádě O, Pavlík V, Šafka V, Lašák P, Pravdová L et al. Telemedicína v léčbě obezity. MMSL 2020; 89 (2): 74-79.

12. Wang C-C, Chao J-K, Wang M-L, Yang Y-P, Chien C-S, Lai W-Y et al. Care for Patients with Stroke During the COVID-19 Pandemic: Physical Therapy and Rehabilitation Suggestions for Preventing Secondary Stroke. J Stroke Cerebrovasc Dis 2020; 29 (11): 105182.

13. Hainer V, Kunešová $M$, Taxová-Braunerová $R$, Zamrazilová $H$, Aldhoon-Hainerová I. Two current pandemics: Obesity and covid-19. Prakt Lek 2020; 100 (4): 159-163.

14. Wilson M, Hull J, Rogers J, Pollock N, Dodd M, Haines J et al. Cardiorespiratory considerations for return-to-play in elite athletes after COVID-19 infection: a practical guide for sport and exercise medicine physicians. Brit J Sport Med 2020; 54.

15. Thomas P, Baldwin C, Bissett B, Boden I, Gosselink R, Granger CL et al. Physiotherapy management for COVID-19 in the acute hospital setting: clinical practice recommendations. J Physiother 2020; 66 (2): 73-82.

16. Kim SY, Kumble S, Patel B, Pruski AD, Azola A, Tatini AL et al. Managing the Rehabilitation Wave: Rehabilitation Services for COVID-19 Survivors. Arch Phys Med Rehabil [Internet]. 22. september 2020 [cit 25. október 2020]; Available at: https: //www.ncbi.nlm.nih.gov/pmc/articles/ PMC7506328/

17. Li Z, Zheng C, Duan C, Zhang Y, Li Q, Dou Z et al. Rehabilitation needs of the first cohort of post-acute COVID-19 patients in Hubei, China. Eur J Phys Rehabil Med 2020; 56 (3): 339-344.

18. Sheehy LM. Considerations for Postacute Rehabilitation for Survivors of COVID-19. JMIR Public Health Surveill [Internet]. 08. máj 2020 [cit 25. október 2020]; 6 (2). Available at: https: //www.ncbi.nlm.nih.gov/ pmc/articles/PMC7212817/

19. Li J. Rehabilitation management of patients with COVID-19: lessons learned from the first experience in China. Eur J Phys Rehab Med 2020; 56 (3): 335-338.

20. Brugliera L, Spina A, Castellazzi P, Cimino P, Tettamanti A, Houdayer E et al. Rehabilitation of COVID-19 patients. J Rehabil Med 2020; 52 (4): jrm00046.

21. Kováčiková Z, Neumannová K, Sarvestan J, Zemková E, Janura M. Sit-to-stand test in fall prediction in elderly adults: What are the options of use in clinical practice? Prakt Lek 2020; 100 (2): 78-82.

22. Michalčíková T, Neumannová K. The occurrence of balance disorders in patients with chronic obstructive pulmonary disease. Rehab Fyzik Lek 2019; 26 (2): 61-67.

23. Benuš P, Kovác J, Gurin B, Líška D. Effect of special breathing exercises on performance and their potential use in rehabilitation. Rehabilitacia 2019; 56 (3): 175-185.
24. Siegerink B, Boon D, Barco S, Klok E. The Post-COVID-19 Functional Status (PCFS) Scale: a tool to measure functional status over time after COVID-19. 06. máj 2020 [cit 25. október 2020]; Available at: https: //osf.io/qgpdv/

25. Petríková Rosinová I, Shtin Banarpvá P, Korcová J. Nordic Walking als geeignete Heilaktivität bei den Patienten mit der Diagnose Diabetes Mellitus. Rehabilitacia 2018; 55 (2): 128-133.

26. Novák J. The importance of gait as the most natural physical activity in the human life style. Prakt Lek 2018; 98 (4): 158-165.

27. Roman-Belmonte JM, De la Corte-Rodriguez H, Rodriguez-Merchan EC, Muñoz-De la Torre E, Vazquez-Sasot A. Strengthening with Blood Flow Restriction: Can it be a Useful Option in the Rehabilitation of Patients with Coronavirus? Arch Bone Jt Surg 2020; 8 (4): 553-536.

28. Michalicka V, Pohnan R. Brain energetic demands during cognitive activities in relation to aerobic load. Military Med Sci Lett 2019; 88 (4): 159-165.

29. Sciriha A, Lungaro-Mifsud S, Fsadni P, Scerri J, Montefort S. Pulmonary Rehabilitation in patients with Interstitial Lung Disease: The effects of a 12-week programme. Respir Med 2019; 146: 49-56.

30. Polastri M, Nava S, Clini E, Vitacca M, Gosselink R. COVID-19 and pulmonary rehabilitation: preparing for phase three. Eur Respir J [Internet]. 25. jún 2020 [cit 25. október 2020]; 55 (6). Available at: https: // www.ncbi.nlm.nih.gov/pmc/articles/PMC7401308/

31. Demeco A, Marotta N, Barletta M, Pino I, Marinaro C, Petraroli A et al. Rehabilitation of patients post-COVID-19 infection: a literature review. J Int Med Res [Internet]. 25. august 2020 [cit 25. október 2020]; 48 (8). Available at: https: //www.ncbi.nlm.nih.gov/pmc/articles/ PMC7450453/

32. Celko J, Guth A, MaSan J, Malay M. Effects of slow diaphragmatic breathing. Rehabilitacia 2019; 56 (4): 275-287.

33. Heffernan KS, Ranadive SM, Jae SY. Exercise as medicine for COVID-19: On PPAR with emerging pharmacotherapy. Med Hypotheses 2020; 143: 110197.

34. da Silveira MP, da Silva Fagundes KK, Bizuti MR, Starck É, Rossi RC, de Resende E Silva DT. Physical exercise as a tool to help the immune system against COVID-19: an integrative review of the current literature. Clin Exp Med 2020.

35. Liu K, Zhang W, Yang Y, Zhang J, Li Y, Chen Y. Respiratory rehabilitation in elderly patients with COVID-19: A randomized controlled study. Complement Ther Clin Pract 2020; 39: 101166.

36. Shan MX, Tran YM, Vu KT, Eapen BC. Postacute inpatient rehabilitation for COVID-19. BMJ Case Rep [Internet]. 18. august 2020 [cit 24. október 2020]; 13 (8). Available at: https: //www.ncbi.nlm.nih.gov/ pmc/articles/PMC7437681/

37. Liu K, Chen Y, Wu D, Lin R, Wang Z, Pan L. Effects of progressive muscle relaxation on anxiety and sleep quality in patients with COVID-19. Complement Ther Clin Pract 2020; 39: 101132.

Received November 30, 2020. Accepted January 7, 2021. 